\title{
在 $\mathrm{Nd}_{2-x} \mathrm{Sr}_{x} \mathrm{NiO}_{4 \pm \lambda}$ 系催化剂上 $\mathrm{NO}$ 分解反应的研究 ${ }^{*}$
}

赵 震 杨向光 吴 越 $* *$

(中国科学院长春应用化学研究所, 长春 130022)

\section{关链词 $\mathrm{Nd}_{2-\mathrm{x}} \mathrm{Sr}_{x} \mathrm{NiO}_{4 \pm \mathrm{A}}$ 系催化剂 $\mathrm{NO}$ 分解}

氮氧化物 $\left(\mathrm{NO}_{x}\right)$ 是严重的空气污染物, 是产生酸雨的主要来源之一. 消除 $\mathrm{NO}_{x}$ 已成为目 前国际上环保化学中最为关键的问题之一. 目前, 世界各国都在大力开发新的消除 $\mathrm{NO}_{x}$ 的方 法, 其中最令人注意的有 $\mathrm{NO}$ 直接分解和寻找可取代 $\mathrm{NH}_{x}$ 的还原剂 ${ }^{[1]}$. 根据已报道的文献 看, 最有希望的 NO 直接分解催化剂除了贵金属 Pd, Pt 之外有 Cu-ZSM-5 分子篮、含 Cu 的 超导体 $\mathrm{Y}-\mathrm{Ba}-\mathrm{Cu}-\mathrm{O}$ 和含 $\mathrm{Co}$ 的 $\mathrm{ABO}_{x}$ 复合氧化物 ${ }^{[2 \sim 4]}$. 把 $\mathrm{A}_{2} \mathrm{BO}_{4}$ 型复合氧化物应用于 $\mathrm{NO}$ 分 解反应的报道很少, 本文首次在 $\mathrm{NO}$ 分解反应中系统研究了 $\mathrm{Ln}_{2-x} \mathrm{Sr}_{x} \mathrm{MO}_{4}(\mathrm{Ln}=\mathrm{La}, \mathrm{Pr}, \mathrm{Nd}, \mathrm{Sm}$, $\mathrm{Gd} ; \mathrm{M}=\mathrm{Cu}, \mathrm{Co}, \mathrm{Ni})$ 系复合氧化物的催化作用, 发现含 $\mathrm{Ni}$ 的 $\mathrm{Nd}_{2-x} \mathrm{Sr}_{x} \mathrm{NiO}_{4}(x=0.0 \sim 1.0)$ 系 催化剂有较高的 NO 分解催化活性, 并提出了可能的反应机理.

\section{1 实验方法}

催化剂的制备、表征和 $\mathrm{Ni}$ 的价态分析见文献[5]. NO 分解反应在固定床流动反应体系中 进行, 反应管为内径 $6 \mathrm{~mm}$ 的石英管, 催化剂粒度为 $40 \sim 80$ 目, 催化剂用量 $0.5 \mathrm{~g}$, 空速为 $2800 \mathrm{~h}^{-1}$. 采用英国产的 Pyc Pan 气相色谱分析检测 NO 的分解率, $0.5 \mathrm{~nm}$ 分子篮为色谱 柱, 用于分离 $\mathrm{NO}, \mathrm{O}_{2}, \mathrm{~N}_{2}$. 由于反应活性测试在较高温度下 $(773 \sim 1173 \mathrm{~K})$ 进行, $\mathrm{N}_{2} \mathrm{O}$ 很难 存在, 因此未能从 Parapork $Q$ 柱检测到 $\mathrm{N}_{2} \mathrm{O}$. 催化剂活性按 $\mathrm{NO}$ 转化率和 $\mathrm{N}_{2}$ 的选择性来 评价.

\section{2 结果与讨论}

\section{$2.1 \mathrm{Ln}_{2-x} \mathrm{Sr}_{x} \mathrm{MO}_{4}$ 系催化剂的 $\mathrm{NO}$ 分解活性}

XRD 结构分析表明制得的 $\mathrm{Ln}_{2-x} \mathrm{Sr}_{x} \mathrm{MO}_{4}(\mathrm{Ln}=\mathrm{La}, \mathrm{Pr}, \mathrm{Nd}, \mathrm{Sm}, \mathrm{Gd} ; \mathrm{M}=\mathrm{Cu}, \mathrm{Co}, \mathrm{Ni})$ 系 复合氧化物的均属 $\mathrm{K}_{2} \mathrm{NiF}_{4}$ 结构, 在 $\mathrm{Nd}_{2-x} \mathrm{Sr}_{x} \mathrm{NiO}_{4 \pm \lambda}$ 系催化剂中 $x=0.0$ 的 $\mathrm{Nd}_{2} \mathrm{NiO}_{4}$ 为单斜结 构, 随 $\mathrm{Sr}$ 掺人后, 使对称性提高, 由单斜转变为四方. 它们的 NO 分解活性数据列于表 1 中. 为了对比,表中还列出一些贵金属和 $\mathrm{ABO}_{x}$ 型复合氧化物的催化活性. 从表 1 可见, 在这些 $\mathrm{A}_{2} \mathrm{BO}_{4}$ 型复合氧化物中未取化的 $\mathrm{Ln}_{2} \mathrm{MO}_{4}$ 催化剂的 $\mathrm{NO}$ 分解活性都很低, 加人 $\mathrm{Sr}$ 取代 $\mathrm{Ln}$ 后 的 $\mathrm{LnSrMO}_{4}(\mathrm{Ln}=\mathrm{La}, \mathrm{Nd} ; \mathrm{M}=\mathrm{Cu}, \mathrm{Co}, \mathrm{Ni})$ 催化剂的 $\mathrm{NO}$ 分解活性大幅度提高, 其中

1994-11-03 收稿, 1995-05-03 收修改稿

*国家自然科学基金资助项目

**联系人 
表 $1 \mathrm{Ln}_{2-x} \mathrm{Sr}_{x} \mathrm{MO}_{4 \pm \lambda}(x=0,1)$ 系催化剂的 $\mathrm{NO}$ 分解活性

\begin{tabular}{|c|c|c|c|c|c|c|c|}
\hline $\begin{array}{c}\text { 催化剂 } \\
\left(\mathrm{K}_{2} \mathrm{NiF}_{4}\right)\end{array}$ & $\begin{array}{c}\text { 反应 } \\
\text { 温度/ }{ }^{\circ} \mathrm{C} \\
\end{array}$ & $\begin{array}{c}\text { NO- 转化 } \\
\text { 率/\% }\end{array}$ & $\begin{array}{c}\mathrm{N}_{2}-\text { 选择 } \\
\text { 性/\% }\end{array}$ & 催化剂 & $\begin{array}{c}\text { 反应 } \\
\text { 温度/C }\end{array}$ & $\begin{array}{c}\text { NO- 转化 } \\
\text { 率/\% } \\
\end{array}$ & $\begin{array}{c}\mathrm{N}_{2} \text { - 选择 } \\
\text { 性/\% }\end{array}$ \\
\hline $\mathrm{La}_{2} \mathrm{CuO}_{4}$ & 800 & 11.51 & 8.21 & \multirow{2}{*}{$\begin{array}{c}\mathrm{La}_{0,9} \mathrm{Ca}_{0,1} \mathrm{MnO}_{3} \\
\left(\mathrm{ABO}_{3}\right)\end{array}$} & 800 & 38.80 & 17.62 \\
\hline $\mathrm{Pr}_{2} \mathrm{CuO}_{4}$ & 800 & 13.48 & 9.60 & & 850 & 61.77 & 35.29 \\
\hline $\mathrm{Nd}_{2} \mathrm{CuO}_{4}$ & 800 & 9.04 & 8.74 & \multirow{2}{*}{$\begin{array}{c}\mathrm{La}_{0,9} \mathrm{Ce}_{0,1} \mathrm{CoO}_{3} \\
\left(\mathrm{ABO}_{3}\right)\end{array}$} & 800 & 7.35 & 5.20 \\
\hline $\mathrm{Sm}_{2} \mathrm{CuO}_{4}$ & 800 & 7.70 & 6.23 & & 850 & 8.16 & 15.46 \\
\hline $\mathrm{Gd}_{2} \mathrm{CuO}_{4}$ & 800 & 5.95 & 5.50 & \multirow[t]{2}{*}{$20 \% \mathrm{Pt} /$ 石棉 } & 800 & 65.10 & 44.70 \\
\hline $\mathrm{Nd}_{2} \mathrm{NiO}_{4}$ & 800 & 10.96 & 9.22 & & 850 & 76.54 & 45.55 \\
\hline \multirow[t]{2}{*}{$\mathrm{LaSrCuO}_{4}$} & 800 & 17.00 & 30.23 & \multirow[t]{2}{*}{$10 \% \mathrm{Pt} /$ 石棉 } & 800 & 43.03 & 29.18 \\
\hline & 850 & 34.29 & 36.96 & & 850 & 48.55 & 31.69 \\
\hline $\mathrm{LaSrCoO}_{4}$ & 800 & 10.52 & 16.87 & $10 \% \mathrm{Pd} /$ 石棉 & 800 & 78.87 & 45.41 \\
\hline & 850 & 20.31 & 24.55 & & 850 & 84.81 & 47.75 \\
\hline \multirow[t]{4}{*}{$\mathrm{NaSrNiO}_{4}$} & 800 & 37.40 & 42.34 & & & & \\
\hline & 850 & 60.50 & 53.90 & & & & \\
\hline & 900 & 77.60 & 56.80 & & & & \\
\hline & $900^{\mathrm{a})}$ & 82.30 & 56.60 & & & & \\
\hline
\end{tabular}

a) 空速减半

$\mathrm{NdSrNiO}_{4}$ 催化剂的 $\mathrm{NO}$ 分解活性最高, 与贵金属 $\mathrm{Pt}$ 相近.

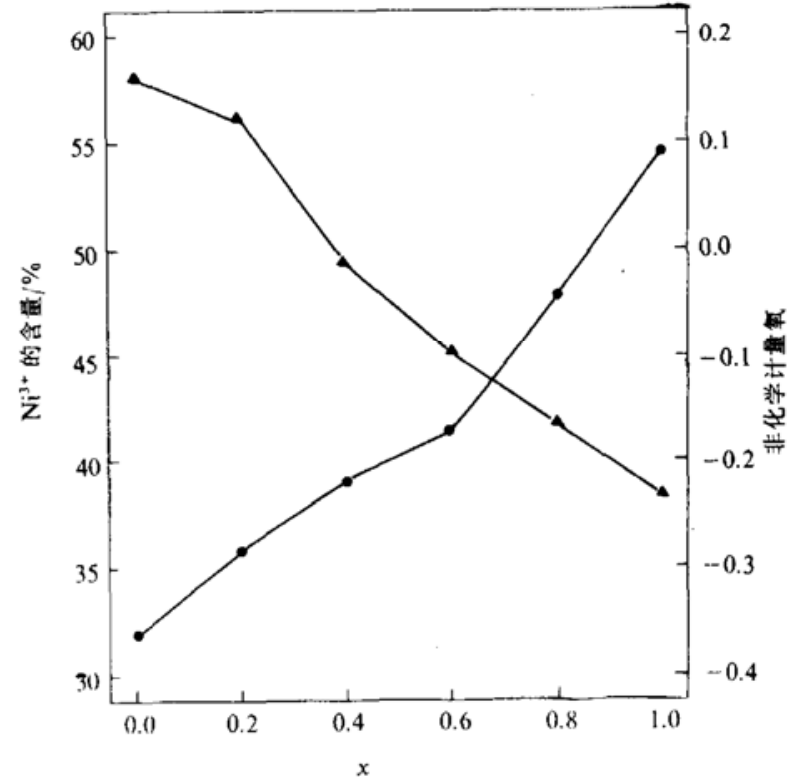

图 I $\mathrm{Nd}_{2}, \mathrm{Sr}_{x} \mathrm{NiO}_{4+i}$ 系催化剂中 $\mathrm{Ni}^{3+}$ 的含量, 非化学 计量氧 $\lambda$ 与 $x$ 的关系

- 为 $\mathrm{Ni}^{31}$ 的含量, $\Delta$ 为非化学计量氧 $\lambda$

\section{$2.2 \mathrm{Nd}_{2} \mathrm{Sr}_{x} \mathrm{NiO}_{4 \pm \lambda}(x=0.0 \sim 1.0)$ 的 缺 陷结构}

利用磺量法, 测定了样品中变价 $\mathrm{Ni}$ 离子的平均价态和 $\mathrm{Ni}^{3+}$ 的含量, 并由此 计算出非化学计量氧 $(\lambda)$. 其变化关系 如图 1 所示. 当低价的 $\mathrm{Sr}^{2+}$ 取代高价 的 $\mathrm{Nd}^{3+}$ 之后, 根据电荷平衡原理 ${ }^{[9]}$, 既 可以使部分 $\mathrm{Ni}$ 离子的价态升高 $\left(\mathrm{Ni}^{2+} \rightarrow \mathrm{Ni}^{3+}\right): \mathrm{Nd}_{2-x} \mathrm{Sr}_{x} \mathrm{Ni}_{1-x}^{2+} \mathrm{Ni}_{x}^{3+} \mathrm{O}_{4}$ $\rightarrow \mathrm{Nd}_{2} \mathrm{NiO}_{4}+x \mathrm{Nd}^{3+}\left|\mathrm{Sr}^{2+}\right|^{\prime}+x \mathrm{Ni}^{2+}\left|\mathrm{Ni}^{3+}\right|^{\cdot}$ 可也产生氧空位:

$\mathrm{Nd}_{2-x} \mathrm{Sr}_{x} \lambda(\mathrm{Vo}) \mathrm{Ni}^{2+} \mathrm{O}_{4-\lambda} \rightarrow \mathrm{Nd}_{2} \mathrm{NiO}_{4}+$ $x \mathrm{Nd}^{3+}\left|\mathrm{Sr}^{2+}\right|^{\prime}+\lambda|\mathrm{O}|^{*} \quad \lambda=1 / 2 x$,

实际上二者常常是同时存在的. $\mathrm{Ni}^{3+}$ 的 含量随 $x$ 的增加而单调增加, 在 $0.0 \leqslant$ $x<0.4$ 范围内 $\mathrm{Ni}^{3+}$ 含量高于理论值, 出 现氧过剩 $(\lambda \geqslant 0)$. 氧过剩的存在形式可 能有两种, 一种是氧离子以随机形式存

在于唱格中, 另一种是出现阳离子空位. $\mathrm{Ni}-\mathrm{O}$ 八面体较稳定, 不易出现 $\mathrm{Ni}$ 的空位, 所以有 可能出现 $\mathrm{La}$ 的缺位. 由于 $\mathrm{O}^{2-}$ 的离子半径较大, 以间隙离子存在于晶格中较为困难, 因此, 后一种形式出现的可能性较大, 在 $0.4 \leqslant x \leqslant 1.0$ 范围内 $\mathrm{Ni}^{3+}$ 含量低于理论值, 出现氧缺陷, 且 随 $x$ 的增加氧缺陷含量增加. 


\section{$2.3 \mathrm{Nd}_{x-x} \mathrm{Sr}_{x} \mathrm{NiO}_{4 \pm \lambda}(x=0.0 \sim 1.0)$ 系复合氧化物的红外光谱分析}

$\mathrm{Nd}_{2-x} \mathrm{Sr}_{x} \mathrm{NiO}_{4 \pm \lambda}$ 系催化剂的红外光谱的结果支持了上述 XRD 结构分析结果. 从图 2 可 以看出, 在 $x=0.0 \sim 1.0$ 范围内, 都在 $500 \mathrm{~cm}^{-1}$ 附近有代表 $\mathrm{A}_{2} \mathrm{BO}_{4}$ 型复合氧化物的强特征吸收

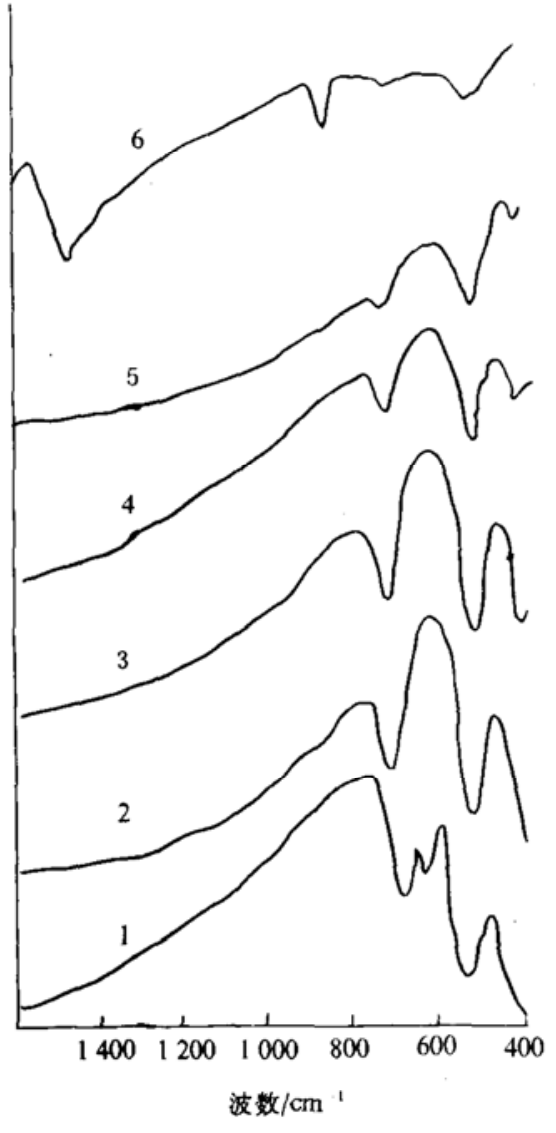

图 $2 \mathrm{Nd}_{2-x} \mathrm{Sr}_{x} \mathrm{NiO}_{4 \pm \lambda}$ 系催化剂的红外证橧 $1 \sim 6$ 的 $x$ 为 $0.0,0.2,0.4,0.6,0.8,1.0$

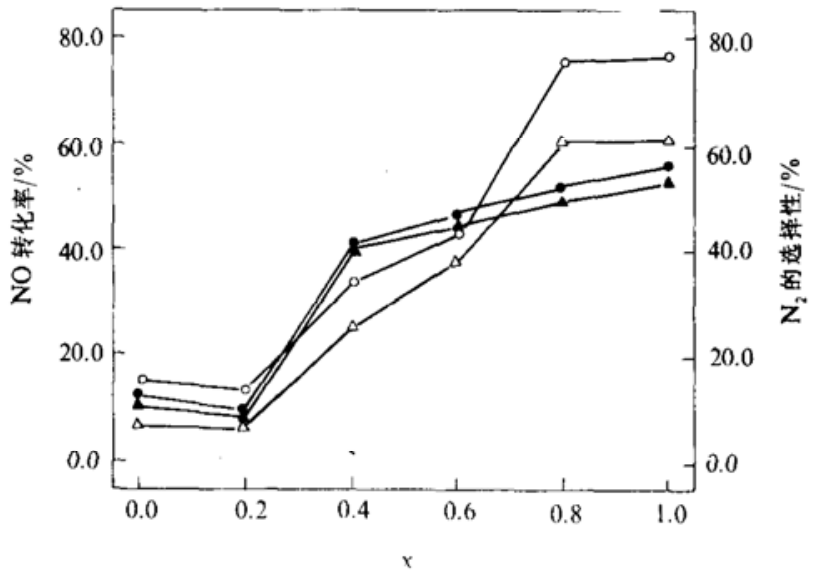

图 $3 \mathrm{Nd}_{2-x} \mathrm{Sr}_{x} \mathrm{NiO}_{4 \pm \lambda}$ 系催化剂的 $\mathrm{NO}$ 分解活性 空速为 $2800^{-1}$; 原料气中 NO 含量 $1.5 \%, \mathrm{He}$ 为稀释气, $\Delta$ 为 $8500^{\circ} \mathrm{C}$ 反应时, $\mathrm{N}_{2}$ 的选择性, -为 $900^{\circ} \mathrm{C}$ 反应时, $\mathrm{N}_{2}$ 的选择性, $\triangle$ 为 $850^{\circ} \mathrm{C}$ 反 应时, $\mathrm{NO}$ 的转化率, $O$ 为 $900^{\circ} \mathrm{C}$ 反应时, $\mathrm{NO}$ 的转化率

带 ${ }^{[6]}$, 表明在 $x=0.0 \sim 1.0$ 范围内生成了 $\mathrm{A}_{2} \mathrm{BO}_{4}$ 结构 的复合氧化物. 对于 $x=0.0$ 的 $\mathrm{Nd}_{2} \mathrm{NiO}_{4}$ 在 $680 \mathrm{~cm}^{-1}$ 附近出现的吸收䢃裂, 说明 $\mathrm{Nd}_{2} \mathrm{NiO}_{4}$ 呈单斜结构, 对称性较低. 随 $\mathrm{Sr}$ 的掺人, 样品由单斜转变为四 方, 对称性提高, 因此, 在 $680 \mathrm{~cm}^{-1}$ 附近的谱峰䢃 裂消失, 且随 $x$ 的增加向高频方向移动, 这可能是 由于 $\mathrm{A}$ 位 $\mathrm{Sr}$ 的掺杂, 对 $\mathrm{a}-\mathrm{b}$ 平面上的 $\mathrm{Ni}-\mathrm{O}_{\mathrm{I}}$ 键作 用加强,而 $510 \mathrm{~cm}^{-1}$ 的谱峰则随 $\mathrm{x}$ 的增加而向低频 方向移动, 这是由于 $\mathrm{Sr}$ 的掺人减弱了 $\mathrm{Nd}-\mathrm{O}_{11}-\mathrm{Ni}$ 键的作用. 值得注意的是, 对于 $x=1.0$ 的样 品, 在 $850 \mathrm{~cm}^{-1}$ 和 $1440 \mathrm{~cm}^{-1}$ 附近处出现吸收带, 它们归属于 $\mathrm{SrCO}_{3}$ 的特征吸收带. 由于 $\mathrm{XRD}$ 物相分析并未检测到 $\mathrm{SrCO}_{3}$, 样品的表面仅存在少量的 $\mathrm{SrCO}_{3}$. 这可能由于 $\mathrm{Sr}$ 大量掺人 时, 有极少量未进人 $\mathrm{Nd}_{2} \mathrm{NiO}_{4}$ 晶格所致.

\section{$2.4 \mathrm{Nd}_{2-x} \mathrm{Sr}_{\mathrm{x}} \mathrm{NiO}_{4 \pm \lambda}$ 系催化剂上 $\mathrm{NO}$ 分解反应的催化性能}

$\mathrm{Nd}_{2-x} \mathrm{Sr}_{x} \mathrm{NiO}_{4 \pm \lambda}(x=0.0 \sim 1.0)$ 系催化剂的 $\mathrm{NO}$ 分解活性变化规律如图 3 所示.

将图 3 和图 1 相对照可以发现, 在 $0.0 \leqslant x \leqslant 0.2$ 区间内为氧过剩, $\mathrm{NO}$ 分解活性很低, 虽然 $\mathrm{Sr}$ 的少量掺人使氧过剩有所降低, 但晶体的对称性提高, $\mathrm{NiO}_{6}$ 八面体的畸变减小, 这样不利 于 $N O$ 分子的吸附与活化. 因此,少量 $\operatorname{Sr}(x=0.2)$ 的掺人活性非但不提高反而稍有降低. 在 $0.4 \leqslant x \leqslant 0.8$ 区间内, 氧含量低于理论值, 存在氧缺陷, 并且随 $x$ 的增加氧缺陷含量的增加, $\mathrm{NO}$ 分解活性急剧增高. 对于 $x=0.4$ 的样品由氧过剩变为氧缺陷, 它的氧缺陷含量仅为 $0.06 \%$, 而 NO 分解活性增加幅度较大. 在 $0.8 \leqslant x \leqslant 1.0$ 区间内, NO 分解活性都较高, 但随 $x$ 的增大 NO 
分解活性变化不大.

由上述分析可以看出, $\mathrm{NO}$ 分解与氧空位直接相关, 而与 $\mathrm{Ni}^{3+}$ 含量不呈顺变关系. 氧空位 在 $\mathrm{NO}$ 分解反应中起着重要作用; $\mathrm{NO}$ 和 $\mathrm{O}_{2}$ 的电子构型接近, 易吸附氧的氧空位对 NO 的吸 附也将十分有利; 氧空位的存在增大了晶格氧的活动性,有利于活性位再生循环的进行.

综合上述分析认为, 在 $\mathrm{Nd}_{2-x} \mathrm{Sr}_{x} \mathrm{NiO}_{4 \pm \lambda}$ 系催化剂上 $\mathrm{NO}$ 分解反应的可能机理为: $\mathrm{NO}$ 分 子首先吸附在 $\mathrm{Ni}^{2+}$ 邻近的氧空位上, $\mathrm{Ni}^{2+}$ 将电子转给 $\mathrm{NO}$ 分子使 $\mathrm{NO}$ 分子的 $\mathrm{N}-\mathrm{O}$ 键弱 化, 从而使 NO 分解.

$$
\begin{gathered}
\mathrm{Ni}^{2+}+\mathrm{Vo}+\mathrm{NO} \rightarrow\left[\mathrm{Ni}^{3+}-\mathrm{Vo}(\mathrm{NO})^{-}\right] \rightarrow \mathrm{Ni}^{3+}+\left[\mathrm{O}^{-}\right]+1 / 2 \mathrm{~N}_{2} \\
{\left[\mathrm{O}^{-}\right]+\mathrm{Ni}^{3+} \rightarrow \mathrm{Ni}^{2+}+\mathrm{Vo}+1 / 2 \mathrm{O}_{2} .}
\end{gathered}
$$

\section{参考 文 献}

1 吴 越. 催化研究的新机遇一环保催化. 科技导报, 1994, 5:57

2 Iwamoto M, Yahiro H, Mine Y et al. Exœssively copper ion-exchanged ZSM-5 zeolites as highly active catalysts of direct decomposition of nitrogen monoxide. Chem Lett, 1989, 213

3 Misoon M, Lombardo E A. Perowskites. Chapter 6.2. elimination of pollutant gases-oxidation of $\mathrm{CO}$ reduction and decomposition of NO. Catal Today, 1990, 8(2): 246

4 Teraoka Y, Fukuda H, Kagawa S. Catalytic activity of perovskite-type oxides for the direct decomposition of nitrogen monoxide. Chem Lett, 1990, $1 \sim 4$

5 Yue W, Tao Y, Bo-sheng D et al. A comparative studty on perowkite-type mixed oxided catalysts $\mathrm{A}^{\prime}{ }_{x} \mathrm{~A}_{1-x} \mathrm{BO}_{3-\lambda}$ $\left(\mathrm{A}^{\prime}=\mathrm{Ca}, \mathrm{Sr}, \mathrm{A}=\mathrm{La}, \mathrm{B}=\mathrm{Mn}, \mathrm{Fe}, \mathrm{Co}\right)$ for $\mathrm{NH}_{3}$ oxidation. $\mathrm{J}$ Catal, 1989, 120:88

6 Odier P, Leblanc M, Leblank C J. Structurral characterization of an orthorhombic form of $\mathrm{La}_{2} \mathrm{NiO}_{4}$. Mater Res Bull, Bull, 1986, 21(7): 787 